\title{
Mixobiota de Floresta Atlântica: novas referências de Physarales para o Estado da Paraíba, Nordeste do Brasil ${ }^{1}$
}

\author{
ANTÔNIA AURELICE AURÉLIO COSTA ${ }^{2,4}$, INALDO DO NASCIMENTO FERREIRA ${ }^{3}$, \\ MARIA DE FÁTIMA DE ANDRADE BEZERRA² e LAISE DE HOLANDA CAVALCANTI ${ }^{2}$
}

(recebido: 17 de junho de 2010; aceito: 14 de abril de 2011)

\begin{abstract}
Atlantic Forest myxomycetes biota: new records of Physarales for the state of Paraíba, Northeast Brazil). The order Physarales encompasses the families Elaeomyxaceae, Didymiaceae, and Physaraceae, involving 16 genera and approximately 371 species, several of them well represented in the Neotropics. A study on the presence of this order in the Atlantic Forest's myxomycetes biota was carried out in 2005 for the Mata do Pau Ferro Permanent Preservation Area (Área de Preservação Permanente Mata do Pau Ferro - APPMPF), located in the Brejo Paraibano mesoregion (6 $6^{\circ} 58^{\prime} 12^{\prime} \mathrm{S}$ and $35^{\circ} 42^{\prime} 15^{\prime}$ W, 400-650 m.a.s.l., 600 ha). Collections of specimens were undertaken between June and December, encompassing the rainy and dry periods, and exploring different substrates. Representative vouchers of the studied material are deposited in the UFP Herbarium. Among the Physarales found, the genus Fuligo is a new record for the state of Paraíba as are the following species: Diderma hemisphaericum (Bull.) Hornem., Didymium clavus (Alb. \& Schwein.) Rabenh., D. nigripes (Link) Fr. (Didymiaceae), Fuligo septica (L.) F. H. Wigg., Physarum echinosporum Lister, P. pulcherrimum Berk. \& Ravenel and P. viride (Bull.) Pers. (Physaraceae). Descriptions based on the studied material are presented for each species, along with comments and the species geographic distribution in Brazil.
\end{abstract}

Key words - Didymiaceae, montane forest, Neotropics, Physaraceae

RESUMO - (Mixobiota de Floresta Atlântica: novas referências de Physarales para o Estado da Paraíba, Nordeste do Brasil). A ordem Physarales engloba as famílias Elaeomyxaceae, Didymiaceae e Physaraceae, reunindo 16 gêneros e aproximadamente 371 espécies, muitas bem representadas nos Neotrópicos. Um estudo sobre a presença de representantes desta ordem na mixobiota da Floresta Atlântica foi desenvolvido na Área de Preservação Permanente Mata do Pau Ferro, localizada na Mesorregião do Brejo Paraibano (6 $6^{\circ} 8^{\prime} 12^{\prime}$ 'S e 35 $42^{\prime} 15^{\prime}$ W, 400-650 m.s.m., 600 ha). As coletas foram realizadas entre junho e dezembro de 2005, abrangendo o período chuvoso e o de estiagem, explorando diferentes substratos. Exsicatas representativas do material estudado estão depositadas no Herbário UFP. Dentre as Physarales assinaladas, constituem nova referência para a Paraíba o gênero Fuligo e as seguintes espécies: Diderma hemisphaericum (Bull.) Hornem., Didymium clavus (Alb. \& Schwein.) Rabenh., D. nigripes (Link) Fr. (Didymiaceae), Fuligo septica (L.) F. H. Wigg., Physarum echinosporum Lister, $P$. pulcherrimum Berk. \& Ravenel e P. viride (Bull.) Pers. (Physaraceae). São apresentadas descrições de cada espécie, baseadas no material estudado, acompanhadas de comentários e distribuição geográfica no Brasil.

Palavras-chave - Didymiaceae, Mata serrana, Neotropicos, Physaraceae

\section{Introdução}

A classe Myxomycetes está representada no Brasil por três espécies de Ceratiomyxomycetidae, 35 de Stemonitomycetidae e pouco mais de 160 Myxogastromycetidae (Cavalcanti 2002, 2005, MaimoniRodella 2002, Putzke 1996, 2002) o que corresponde

1. Parte da dissertação de mestrado da primeira autora, Programa de Pós-Graduação em Biologia de Fungos, Universidade Federal de Pernambuco, Recife, PE, Brasil.

2. Universidade Federal de Pernambuco, Departamento de Botânica, Laboratório de Myxomycetes, Av. Prof. Moraes Rego s/n, Cidade Universitária, 50.670-901 Recife, PE, Brasil.

3. Programa de Pós-Graduação em Biologia de Fungos, Universidade Federal de Pernambuco, Departamento de Micologia, Centro de Ciências Biológicas, Av. Prof. Moraes Rego s/n, Cidade Universitária, 50.670-901 Recife, PE, Brasil.

4. Autor para correspondência: licebio2002@gmail.com a cerca de $20 \%$ do total de espécies mundialmente conhecido (Lado 2001, 2005-2010).

Dentre as Myxogastromycetidae, as Physarales destacam-se na mixobiota brasileira pelo número e distribuição dos gêneros e espécies, que correspondem a 44\% do total de representantes da subclasse conhecidos para o país. As espécies desta ordem ocupam os mais diferentes ambientes e microhabitats, com registros nas matas úmidas costeiras, nas caatingas, nos diferentes tipos de cerrado e até mesmo em manguezais (Cavalcanti 1996, 2005). Em canaviais, material armazenado em indústrias, parques e jardins públicos ou residenciais, têm sido reportadas espécies de Badhamia, Craterium, Diachea, Diderma, Didymium, Fuligo e Physarum, particularmente na Região Nordeste, onde os estudos têm se concentrado nas últimas décadas (Cavalcanti 1996). 
Physarales é uma das ordens mais numerosas dos Myxomycetes, com 16 gêneros e 371 espécies, porém abrange apenas três famílias: Elaeomyxaceae, Didymiaceae e Physaraceae (Martin et al. 1983).

Elaeomyxaceae se caracteriza pela presença de nódulos de graxa em diferentes estruturas do esporocarpo, localizando-se dentro do pedicelo (quando presente) e algumas vezes na columela, capilício e perídio. Essa família foi inicialmente proposta por Hagelstein (1942), para abrigar as espécies de seu novo gênero Elaeomyxa, baseado em Diachea cerifera G. Lister, da ordem Stemonitales. Keller (1980) transferiu o gênero para Physarales, colocando-o nas Didymiaceae, dando um tratamento semelhante ao efetuado para as espécies de Diachea após a separação das Stemonitales em uma nova subclasse (Martin et al. 1983). Farr \& Keller (1982) validaram a família Elaeomyxaceae proposta por Hagelstein (1942), posição acatada por Martin et al. (1983). Atualmente são conhecidas para a família as duas espécies propostas por Hagelstein (1942), Elaeommyxa cerifera (G. Lister) Hagelst. e E. miyazakiensis (Emoto) Hagelst., e E. australiensis (S.L. Stephenson, G. Moreno \& H. Singer) G. Moreno, H. Singer \& S.L. Stephenson e E reticulospora (Gilert) G. Moreno, H. Singer \& S.L. Stephenson, recentemente transferidas do gênero Lamproderma (Lado 2005-2010), todas sem ocorrência conhecida para a América do Sul até o momento.

Didymiaceae se caracteriza por não incluir cálcio no capilício, apesar do perídio, columela e pedicelo poderem se apresentar densamente calcários (Martin et al. 1983). A família compreende seis gêneros e 178 espécies (Lado 2001, 2005-2010), muitas delas com ampla distribuição mundial, como Didymium nigripes (Link) Fr. e Diderma effusum (Schw.) Morgan. No Brasil, as Didymiaceae estão representadas por Diachea, com três espécies, Diderma, com 10 espécies e Didymium, com 14 espécies, distribuídas em todas as regiões, incluindo o Centro-Oeste, a menos explorada do país (Cavalcanti 2002, Maimoni-Rodella 2002, Putzke 1996, 2002). Novas espécies para a ciência foram descritas, com base em material coletado no Brasil, como Didymium bahiense Gottsb., de ampla distribuição mundial, cuja localidade tipo é o Morro do Chapéu (BA), em ambiente de caatinga (Gottsberger 1968) e Diderma albocolumella A.C.C. Bezerra \& L.H. Cavalc. coletada em fragmento de Floresta Atlântica no Rio Grande do Norte (Bezerra \& Cavalcanti 2010).

Physaraceae se diferencia pela presença de incrustações calcárias em quase todas as estruturas que compõem o esporocarpo, principalmente na rede capilicial. A família compreende 10 gêneros e 196 espécies (Lado 2001, 2005-2010), muitas cosmopolitas, como Fuligo septica (L.) F. H. Wigg., Physarum album (Bull.) Chevall. e Craterium leucocephalum (Pers. ex J. F. Gmel.) Ditmar. No Brasil, as Physaraceae estão representadas por Badhamia, com oito espécies, Badhamiopsis, com uma espécie, Craterium, com quatro espécies, Fuligo, com três espécies, Physarum, com 47 espécies, Willkommlangea, Leocarpus e Physarella, monotípicos. As espécies de Physaraceae têm registros em todas as regiões do país, incluindo o Centro-Oeste, para onde foi descrito Physarum alvoradianum Gottsb., cuja localidade tipo é Alvorada do Norte, Goiás, em ambiente de cerrado (Gottsberger 1968).

Os estudos realizados no Nordeste do Brasil registraram a ocorrência de 21 espécies de Didymiaceae, pertencentes aos gêneros Diachea, Diderma e Didymium e 40 espécies de Physaraceae, distribuídas nos gêneros Badhamia, Badhamiopsis, Craterium, Fuligo, Physarella e Physarum (Cavalcanti 2002, Cavalcanti et al. 2006a). Alguns estados têm a mixobiota melhor conhecida, como a Bahia, com as primeiras coletas realizadas no início do século XX (Torrend 1915), e Pernambuco, cuja mixobiota está sendo estudada desde o final da década de 1940 (Batista 1949). Os primeiros estudos no Piauí foram realizados no final dos anos 1990 e, atualmente, estão registradas para este estado todas as subclasses e ordens de Myxomycetes, com nove famílias e 54 espécies, incluindo 18 espécies de Physarales (Cavalcanti et al. 2006b). Inventário recentemente concluído no Parque Nacional Serra de Itabaiana permitiu os primeiros registros de Myxomycetes para Sergipe, com representação de todas as subclasses e ordens de Myxomycetes, com 27 espécies de Physarales (Bezerra et al. 2008).

Os demais estados do Nordeste se encontram com a mixobiota pouco explorada e apenas quatro espécies são conhecidas para o Maranhão, todas da ordem Physarales, com os registros de Didymium iridis (Ditmar) Fr., Physarum compressum Alb. \& Schwein., P. album e Fuligo septica (Torrend 1915, Silva \& Bezerra 2005). Encontram-se, também, poucos registros para: Ceará, onde foram assinalados quatro gêneros e nove espécies de Physarales por Alves \& Cavalcanti (1996), Cavalcanti \& Putzke (1998); Alagoas, com cinco espécies de Didymiaceae e 16 de Physaraceae (Cavalcanti 2002, Cavalcanti et al. 2006a); Rio Grande do Norte, com 10 espécies de Physarales (Cavalcanti 2002, Bezerra et al. 2007).

A mixobiota paraibana se encontra praticamente inexplorada, sendo conhecida a ocorrência de quatro espécies de Liceales, seis de Trichiales, sete 
de Stemonitales e seis de Physarales, estas últimas representadas por Craterium leucocephalum, Physarella oblonga (Berk. \& M. A. Curtis) Morgan, Physarum. album, P. bogoriense Racib., P. oblatum T. Macbr. e Diderma effusum (Cavalcanti \& Araújo 1985, Cavalcanti \& Marinho 1985, Cavalcanti \& Oliveira 1985, Cavalcanti \& Silva 1985, Cavalcanti 2002). O presente trabalho, desenvolvido no Município de Areia, Mesorregião do Brejo Paraibano, adiciona ao conhecimento da mixobiota paraibana o gênero Fuligo e sete espécies de Physarales, elevando para 13 o número de representantes das Physarales de ocorrência conhecida neste Estado.

\section{Material e métodos}

Área estudada - A Área de Preservação Permanente Mata do Pau Ferro, situada no Município de Areia, na Mesorregião do Brejo Paraibano $\left(6^{\circ} 58^{\prime} 12^{\prime} \mathrm{S}\right.$ e $\left.35^{\circ} 42^{\prime} 15^{\prime} \mathrm{W}\right)$ é uma área de Floresta Ombrófila Densa considerada representativa dos Brejos de altitude nordestinos. Está situada a $5 \mathrm{~km}$ a oeste da sede do Município de Areia, a uma altitude entre 400-600 m, com uma área de cobertura de 600 ha. O clima é ameno, devido ao relevo e direção dos ventos vindos do Atlântico (dista aproximadamente $127 \mathrm{~km}$ do litoral), com temperatura média entre $15-18{ }^{\circ} \mathrm{C}$ no inverno e $22-30{ }^{\circ} \mathrm{C}$ no verão e $1.450 \mathrm{~mm}$ de precipitação pluviométrica anual (Mayo \& Fevereiro 1982).

Coleta e tratamento dos espécimes - Para o inventário das Physarales presentes na mixobiota dessa Área de Preservação de Floresta Atlântica paraibana, foram realizadas coletas em 2005 nos meses chuvosos (junho e agosto) e de estiagem (setembro-dezembro). Em cada ocasião, foram exploradas três trilhas, com extensão total de $7,7 \mathrm{~km}$, nas quais foi pesquisada a presença de esporocarpos em troncos de árvores mortas não identificadas, em pé ou caídos, casca de árvores vivas, necromassa que cobre o solo da floresta (folhas, gravetos, frutos, inflorescências, etc), folhedo aéreo e basidiocarpos. Amostras destes substratos foram coletadas e utilizadas para a preparação de 100 câmaras-úmidas (Schnittler \& Stephenson 2002).

A identificação dos espécimes de Physarales, obtidos em campo e em câmara-úmida, foi realizada com o auxílio de chaves analíticas, a partir de caracteres revelados por análise morfológica ao estereomicroscópio e ao microscópio óptico (Mobin \& Cavalcanti 1999), complementadas por descrições encontradas na literatura especializada, especialmente Martin \& Alexopoulos (1969), Farr (1976) e Nannenga-Bremekamp (1991).

Adotou-se o sistema de classificação de Martin et al. (1983) para gêneros e categorias supragenéricas, a nomenclatura e citação de autores das espécies seguem as indicadas por Lado (2001).

Foi revisada a bibliografia sobre Physarales ocorrentes no Brasil, enfocando-se a distribuição das espécies nos diferentes Estados e ambientes para os quais foram citadas; para cada espécie, indica-se o autor que publicou a primeira referência para o país.

\section{Resultados e discussão}

\section{DIDYMIACEAE}

Diderma Pers. 1794

Diderma hemisphaericum (Bull.) Hornem., Fl. Dan. 33: 13 1829. $\equiv$ Reticularia hemisphaerica Bull. Herb. France, pl. 446 f. 1.1790.

Figura $1 \mathrm{~A}-\mathrm{C}$

Esporângio pedicelado, ereto, 1,00 mm alt. total, esporoteca branca a cinza, discóide, umbilicada levemente na parte superior e fortemente na base; hipotalo concolor ao pedicelo, individualizado; pedicelo subcilíndrico, grosso, 0,63 mm comp., castanho claro; perídio de deiscência circuncisa, duplo, camada interna membranosa, delicada, incolor, camada externa calcária, branca; columela inconspícua; capilício escasso, castanho-violáceo pálido, filamentos dicotômicos, 1,0 $\mu \mathrm{m}$ diâm.; esporada castanho-acinzentado; esporo castanho-violáceo claro sob luz transmitida, globoso, quase liso, 7,14-10,2 $\mu \mathrm{m}$ diâm.

Apenas um espécime foi obtido no presente estudo, desenvolvido em câmara-úmida preparada com folhedo, mas os esporocarpos apresentaram-se muito bem formados e com as características típicas da espécie, a qual é referida pela primeira vez para a Paraíba.

Distribuição: cosmopolita (Martin \& Alexopoulos 1969). No Brasil, ocorre nas Regiões Sudeste (São Paulo, Torrend 1915) e Nordeste, com registros para Bahia (Torrend 1915) e Pernambuco (Farr 1960).

Material examinado: BRASIL.PARAíBA: Areia, Mata do Pau Ferro, Trilha Flores, câmara-úmida 8-VI-2005, esporulação 5-VII-2005, A.A.A. Costa et al. 5 A (UFP 44.764).

Didymium Schrad. 1797

Didymium clavus (Alb. \& Schwein.) Rabenh., Deutschl. Krypt.-Fl. 1: 280. 1844. इ Physarum clavus Alb. \& Schwein., Consp. Fung. Lusat. 96. 1805.

\section{Figura $1 \mathrm{D}-\mathrm{F}$}

Esporângio gregário, pedicelado, ereto, 1,00 mm alt. total, esporoteca discóide, umbilicada na base, branco a cinza; hipotalo membranáceo, circular, castanho; pedicelo robusto, negro, opaco na base e mais claro para o ápice, subcilíndrico, estriado longitudinalmente, 0,50 mm comp.; perídio membranáceo, castanho, coberto com cristais de cálcio; columela discóide, castanho- 

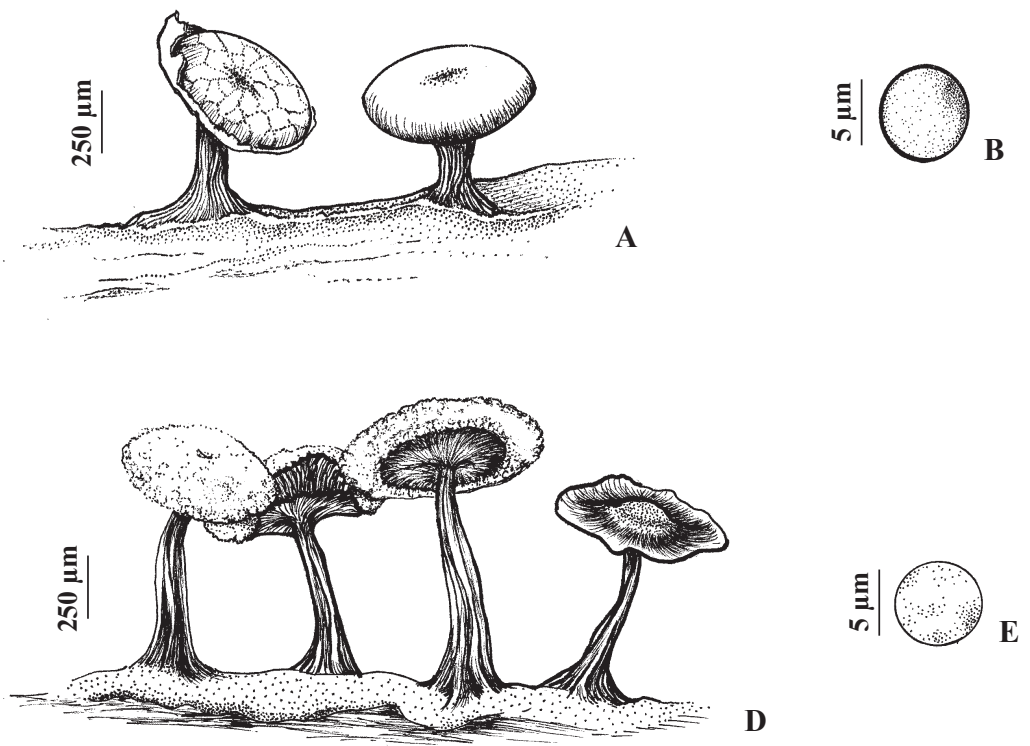

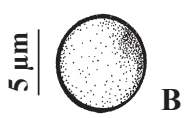

A

D

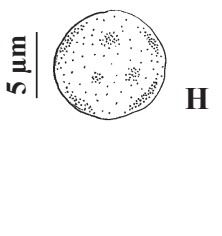

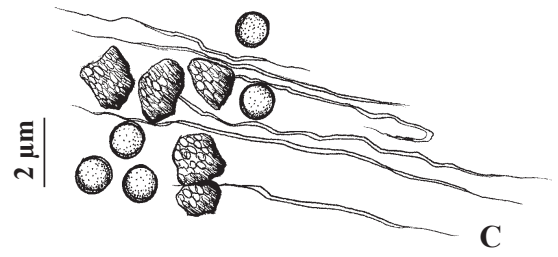
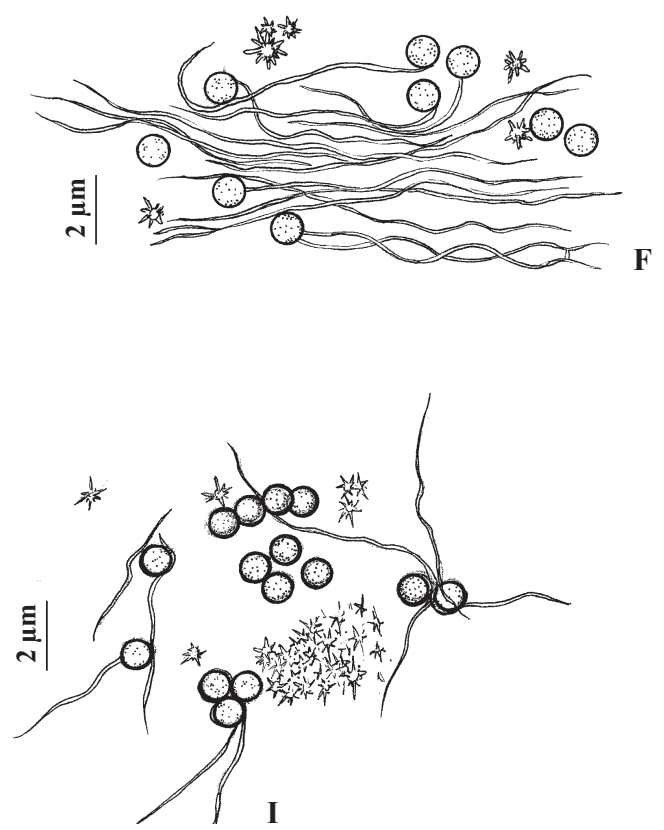

Figura 1. A-C. Diderma hemisphaericum. A. Esporângios. B. Esporo. C. Capilício e esporos. D-F. Didymium clavus. D. Esporângios. E. Esporo. F. Capilício e esporos. G-I. Didymium nigripes. G. Esporângios. H. Esporo. I. Capilício e esporos. (A-C. A.A.A. Costa et al. 5; D-F. A.A.A. Costa et al. 173; G-I. A.A.A. Costa et al. 594).

Figure 1. A-C. Diderma hemisphaericum. A. Sporangia. B. Spore. C. Capillicium and spores. D-F. Didymium clavus. D. Sporangia. E. Spore. F. Capillicium and spores. G-I. Didymium nigripes. G. Sporangia. H. Spore. I. Capillicium and spores (A-C. A.A.A. Costa et al. 5; D-F. A.A.A. Costa et al. 173; G-I. A.A.A. Costa et al. 594).

escuro; capilício esparsamente ramificado, liso, delicado, castanho-púrpura; esporada castanho-escuro; esporo globoso, verrucoso, com grupos de verrugas maiores, castanho-violáceo, 6,12-7,14 $\mu \mathrm{m}$ diâm.

Um único espécime foi obtido no presente estudo, coletado sobre tronco morto caído; os esporângios apresentaram-se muito bem formados e com as características típicas da espécie, a qual é referida pela primeira vez para a Paraíba.

Distribuição: no Brasil, ocorre nas regiões Norte (Roraima, Cavalcanti et al. 1999), Sudeste (São Paulo, Spegazzini 1919), Sul (Rio Grande do Sul, Rodrigues \& Guerrero 1990; Santa Catarina, Bresadola 1896 apud Cavalcanti \& Fortes 1995). No Nordeste, têm registros para Bahia (Torrend 1915), Pernambuco (Farr 1960) e Piauí (Mobin \& Cavalcanti 1999).

Material examinado: BRASIL. PARAíBA: Areia, Mata do Pau Ferro, Trilha das Flores, 26-VIII-2005, A.A.A. Costa et al. 173 (UFP 44.763).

Didymium nigripes (Link) Fr., Syst. Mycol. 3: 119. 1829. 三 Physarum nigripes Link, Ges. Naturf. Freunde Berlin Mag. Neuesten Entdeck. Gesammten Naturk. 3: 27. 1809.

Figura 1 G-I

Esporângio pedicelado, gregário, 0,90-1,40 mm alt. total; esporoteca globosa a subglobosa, fortemente umbilicadanabase, brancoa cinza; hipotalomembranáceo, 
circular, castanho; pedicelo ereto e levemente flexuoso, castanho-enegrecido, estriado longitudinalmente; perídio membranáceo, castanho, brilhante, coberto com cristais de cálcio; columela subglobosa, castanhoescuro, 193,80 $\mu \mathrm{m}$ comp. e 102,00 $\mu \mathrm{m}$ larg.; capilício delicado, castanho-avermelhado; esporada castanha; esporo globoso, verrucoso, castanho-violáceo sob luz transmitida, 7,14-10,20 $\mu \mathrm{m}$ diâm.

Os esporocarpos dos dois espécimes obtidos no presente estudo apresentaram a esporoteca fortemente umbilicada, com a columela castanho-enegrecida típica da espécie, a qual ainda não dispunha de registros para a Paraíba.

Distribuição: cosmopolita (Martin \& Alexopoulos 1969). No Brasil, ocorre nas regiões Norte (Roraima, Cavalcanti et al. 1999), Sudeste (Rio de Janeiro, Klotzsch, 1843 apud Putzke 1996; São Paulo, Sydow \& Sydow 1907), Sul (Rio Grande do Sul, Rodrigues \& Guerrero 1990). No Nordeste, tem registros para Pernambuco (Farr 1960) e Piauí (Mobin \& Cavalcanti 1999).

Material examinado: BRASIL. PARAíBA: Areia, Mata do Pau Ferro, Trilha Boa Vista, 3-VI-2005, A.A.A. Costa et al. 2 (UFP 41.839); Trilha do Cumbe, 6-XI-2005, A.A.A. Costa et al. 594 (UFP 44.539).

\section{PHYSARACEAE}

Fuligo Haller 1768

Fuligo septica (L.) F. H. Wigg., Prim. Fl. Holsat.: 112 1780. $\equiv$ Mucor septicus L., Sp. Pl., ed. 2. 1656. 1763. Figura 2 A-C

Etálio pulvinado, amarelo, 3,00-5,00 $\mathrm{mm}$ alt. e 20,00-45,00 mm comp.; córtex incrustado de cálcio, amarelo; hipotalo branco, bem desenvolvido, irregular, membranáceo, calcário; capilício abundante, filamentos hialinos, nódulos calcários irregulares, amarelos; esporada castanho-enegrecido; esporo globoso, com diminutas verrugas, castanho-pálido sob luz transmitida, 6,12-9,18 $\mu \mathrm{m}$ diâm.

O único espécime obtido no presente estudo apresentou as típicas características desta espécie cosmopolita, com ampla distribuição no Brasil, em ambientes naturais ou antropizados (Santos \& Cavalcanti 1988, 1991). Vários Estados do Nordeste têm registros de $F$. septica, mas nenhuma espécie do gênero havia sido referida para a Paraíba até o presente momento.

Distribuição: cosmopolita (Martin \& Alexopoulos 1969). No Brasil, ocorre nas regiões Norte (Amazonas, Jahn 1904; Roraima, Cavalcanti et al. 1999), Sudeste (Minas Gerais, Torrend 1915; São Paulo, Torrend 1915) e Sul (Paraná, Gottsberger et al. 1992; Rio Grande do Sul, Rodrigues \& Guerrero 1990; Santa Catarina, Hennings 1896 apud Cavalcanti \& Fortes 1995). No Nordeste, têm registros para Alagoas (Cavalcanti et al. 1985), Bahia (Torrend 1915), Ceará (Alves \& Cavalcanti 1996), Pernambuco (Farr 1960) e Maranhão (Silva \& Bezerra 2005).

Material examinado: BRASIL. PARAíBA: Areia, Mata do Pau Ferro, Trilha das Flores, 19-XII-2005, A.A.A. Costa et al. 695 (UFP 42.673).

Physarum Pers. 1794

Physarum echinosporum Lister, J. Bot. 37: 147.1899.

Figura $2 \mathrm{D}-\mathrm{F}$

Plasmodiocarpo branco puro a branco-acinzentado, $1,00 \mathrm{~mm}$ alt. total, usualmente curvado, fortemente comprimido lateralmente; perídio duplo, camada externa fortemente calcária, tipo porcelana, camada interna membranosa, deiscência por uma fissura apical; capilício liso, amarelo-claro, nódulos de cálcio brancos, pequenos, globosos a subglobosos; esporada castanhoescuro; esporo castanho-púrpura, globoso, parede com espinhos bem desenvolvidos que às vezes se unem e formam rugas, 10,20-13,26 $\mu \mathrm{m}$ diâm.

O único espécime obtido é proveniente de material desenvolvido em câmara-úmida montada com folhedo aéreo. A frutificação foi abundante, com esporocarpos bem formados, apresentando as características típicas da espécie. Physarum echinosporum tem registros para o Brasil em áreas antropizadas em Pernambuco e em ambiente natural no Estado de São Paulo. Este é o primeiro registro para a Paraíba e o primeiro em que a espécie é encontrada em ambiente natural no Nordeste do país.

Distribuição: no Brasil, ocorre nas Regiões Sudeste (São Paulo, Hochgesand \& Gottsberger 1996) e Nordeste, com registros apenas para Pernambuco (Cavalcanti 1976).

Material examinado: BRASIL. PARAÍBA: Areia, Mata do Pau Ferro, Trilha Boa Vista, 09-XI-2006, A.A.A. Costa et al. 76 (UFP 44.534).

Physarum pulcherrimum Berk. \& Ravenel, in Berkeley, Grevillea 2: 65. 1873.

Figura 2 G-I

Esporângio pedicelado, globoso, vermelho, 1,00 $\mathrm{mm}$ alt. total; hipotalo irregular, membranáceo, castanho; pedicelo cilíndrico, calcário, fibroso, 0,91 mm comp., enrugado, castanho brilhante, ápice 71,4 $\mu \mathrm{m}$ larg., base 142,8 $\mu \mathrm{m}$ larg.; perídio calcário, membranoso, simples, deiscência irregular, vermelho- 


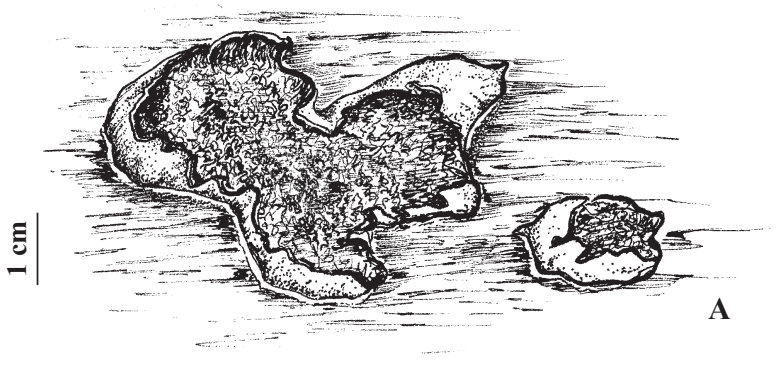

A
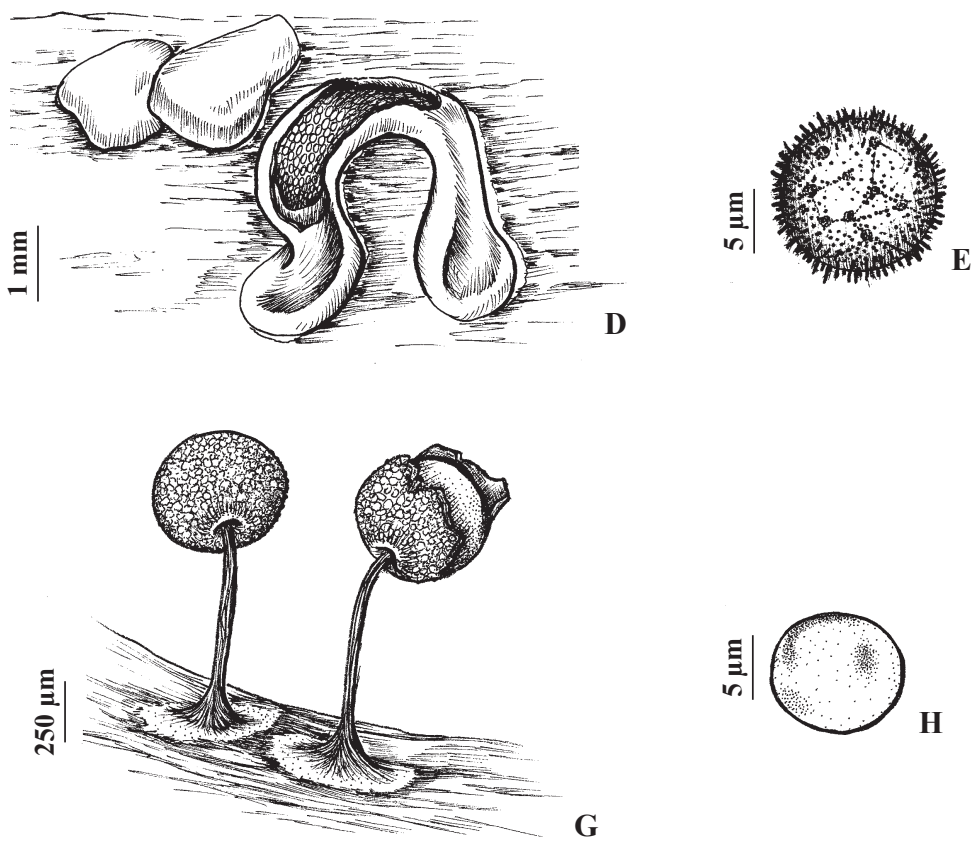
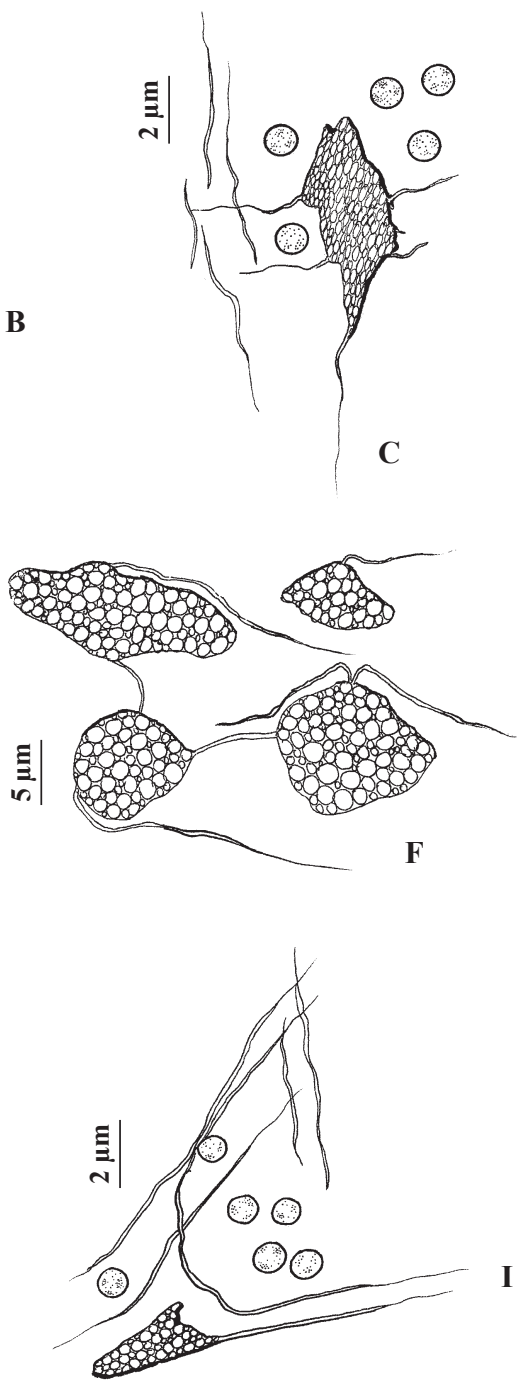

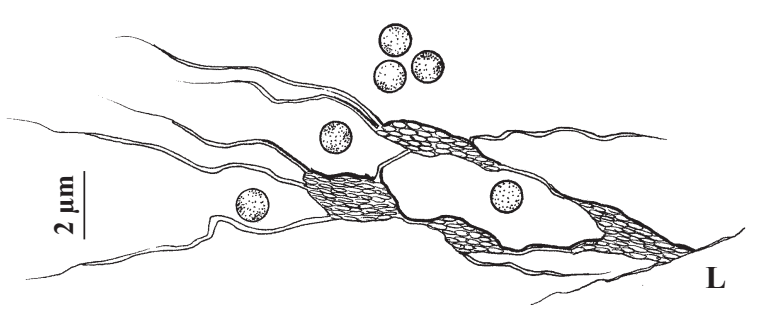

Figura 2. A-C. Fuligo septica. A. Etálio. B. Esporo. C. Capilício e esporos. D-F. Physarum echinosporum. D. Plasmodiocarpos. E. Esporo. F. Capilício. G-I. Physarum pulcherrimum. G. Esporângios. H. Esporo. I. Capilício e esporos. J-L. Physarum viride. J. Esporângios. K. Esporo. L. Capilício e esporos. (A-C. A.A.A. Costa et al. 695; D-F. A.A.A. Costa et al. 76; G-I. M.F.A. Bezerra 505; J-L. A.A.A. Costa et al. 698).

Figure 2. A-C. Fuligo septica. A. Ethalium. B. Spore. C. Capillicium and spores. D-F. Physarum echinosporum. D. Plasmodiocarps. E. Spore. F. Capillicium. G-I. Physarum pulcherrimum. G. Sporangia. H. Spore. I. Capillicium and spores. J-L. Physarum viride. J. Sporangia. K. Spore. L. Capillicium and spores. (A-C. A.A.A. Costa et al. 695; D-F. A.A.A. Costa et al. 76; G-I. M.F.A. Bezerra 505; J-L. A.A.A. Costa et al. 698). 
violáceo sob luz transmitida; columela cônica, atingindo cerca de $1 / 4$ da esporoteca; capilício denso, delicado, filamentos numerosos, nódulos de cálcio grandes, irregulares, normalmente agrupados no centro da esporoteca, vermelhos; esporada castanho-escuro; esporo globoso, minutamente verrucoso e com agrupamento de verrugas, rosa pálido sob luz transmitida, 8,16-9,18 $(10,2) \mu \mathrm{m}$ diâm.

Apenas um espécime foi obtido no presente estudo, apresentando as típicas características de $P$. pulcherrimum, que se assemelha a $P$. roseum Berk. \& Broome, mas se diferencia pelos nódulos de cálcio menores, pela cor do esporocarpo e formato da columela. Este é o segundo registro desta espécie para o país, sendo o primeiro obtido recentemente por Bezerra et al. (2008), no Parque Nacional da Serra de Itabaiana.

Distribuição: No Brasil, tem registro apenas para a região Nordeste (Sergipe, Bezerra et al. 2008)

Material examinado: BRASIL. PARAíBA: Areia, Mata do Pau Ferro, Trilha Boa Vista, 5-XI-2005, A.A.A. Costa et al. 535 (UFP 42.647).

Physarum viride (Bull.) Pers., Ann. Bot. (Usteri) 15: 6. 1795. $\equiv$ Sphaerocarpus viridis Bull., Hist. Champ. France 135. 1791.

Figura $2 \mathrm{~J}-\mathrm{L}$

Esporângio longo-pedicelado, 1,00 mm alt. total, esporoteca subglobosa a lenticular, amarelo-alaranjada; hipotalo irregular, membranáceo, hialino; pedicelo subcilíndrico, calcário, usualmente $3 / 4$ da altura total, castanho escuro na base, 71,40 $\mu \mathrm{m}$ larg., mais claro no ápice, 20,40 $\mu \mathrm{m}$ larg.; perídio membranoso, simples, amarelo, deiscência irregular; capilício denso, ramificado dicotomicamente, nódulos de cálcio fusiformes a irregulares, amarelos; esporada castanho-escuro; esporo globoso, minutamente verrucoso a quase liso, castanho, 7,16-10,20 $\mu \mathrm{m}$ diâm.

Os dois espécimes obtidos apresentaram as características descritas por Martin \& Alexopoulos (1969) para a espécie, que apresenta ampla distribuição no Brasil, em diferentes ecossistemas, mas não havia sido registrada para a mixobiota paraibana.

Distribuição: Cosmopolita (Martin \& Alexopoulos 1969). No Brasil, ocorre nas Regiões Norte (Amazonas, Jahn 1904), Sudeste (Rio de Janeiro, Torrend 1915; São Paulo, Bononi et al. 1981) e Sul (Paraná, Gottsberger et al. 1992; Santa Catarina, Bresadola 1896 apud Cavalcanti \& Fortes 1995). No Nordeste tem registros para Pernambuco (Farr 1960) e Piauí (Mobin \& Cavalcanti 1999).
Material examinado: BRASIL. Paraíba: Areia, Mata do Pau Ferro, Trilha das Flores, 3-VI-2005, A.A.A. Costa et al. 1 (UFP 41.838); 19-XII-2005, A.A.A. Costa et al. 698 (UFP 44.538).

Agradecimentos-Os autores agradecem ao Conselho Nacional de Desenvolvimento Cientifico e Tecnológico (CNPq), pela concessão das bolsas de estudo sobre Myxomycetes no Nordeste do Brasil (processos 133656/2005-5, 140327/2005, 155673/2006-8, 479184/2003-8); a Marlene C. A. Barbosa, curadora do herbário UFP, pelas informações concedidas e material consultado; ao Dr. Leonardo Pessoa Félix, da Universidade Federal da Paraíba, Campus de Areia, pela disponibilidade e apoio para o desenvolvimento desta pesquisa; à DSc Andrea C. Caldas Bezerra, MSc. Alessandra Alencar, Bel. Fabian Tavares, MSc. Glauciane Damasceno, MSc Márcio Rufino, MSc David Lemos e Bel. Leandro Agra, da equipe do Labmix-UFPE, pelo auxílio nos trabalhos de campo e laboratório.

\section{Referências bibliográficas}

ALVES, M.H. \& CAVALCANTI, L.H. 1996. Myxomycetes em palmeiras (Arecaceae). Acta Botanica Brasilica 10:1-7.

BATISTA, A.C. 1949. Três mixomicetes comuns em Pernambuco. Boletim da Secretaria de Agricultura, Indústria e Comércio do Estado de Pernambuco 16: 166-167.

BEZERRA, A.C.C. \& CAVALCANTI, L.H. 2010. Diderma albo-columella (Myxomycetes), a new species in the Brazilian Atlantic Forest. Rodriguésia 61:105-108.

BEZERRA, A.C.C., NUNES, A.T., COSTA, A.A.A., FERREIRA, I.N., BEZERRA, M.F.A. \& CAVALCANTI, L.H. 2007. Mixobiota do Parque Estadual das Dunas de Natal. Revista Brasileira de Biociências 5:30-32.

BEZERRA, M.F.A., BEZERRA, A.C.C., NUNES, A.T., LADO, C. \& CAVALCANTI, L.H. 2008. Mixobiota do Parque Nacional Serra de Itabaiana, SE, Brasil: Physarales. Acta Botanica Brasilica 22: 1044-1056.

BONONI, V.L.R., TRUFEM, S.F.B. \& GRANDI, R.A.P. 1981. Fungos macroscópicos do Parque Estadual das Fontes do Ipiranga, São Paulo, Brasil, depositados no Herbário do Instituto de Botânica. Rickia 9: $37-53$

CAVALCANTI, L.H. 1976. Mixomicetos novos para Pernambuco II. Memórias do Instituto de Biociências, Universidade Federal de Pernambuco, Botânica 4:119.

CAVAlCANTI, L.H. 1996. Myxomycetes. In Pesquisa Botânica Nordestina: progresso e perspectivas (E.V.S.B. Sampaio, S.J. Mayo \& M.R.V. Barbosa, orgs.). Sociedade Botânica do Brasil. Recife. p.37-45. 
CAVALCANTI, L.H. 2002. Biodiversidade e distribuição de mixomicetos em ambientes naturais e antropogênicos no Brasil: espécies ocorrentes nas Regiões Norte e Nordeste. In Biodiversidade, conservação e uso sustentável da flora do Brasil (E.L.L.A. Araujo, A.N. Moura, E.V.S.B. Sampaio, L.M.S. Gestinari \& J.M.T., Carneiro, eds.) Universidade Federal Rural de Pernambuco, Sociedade Botânica do Brasil. Recife. p.209-216.

CAVALCANTI, L.H. 2005. Mixomicetos do Brasil: situação atual. In Anais do V Congresso Latino Americano de Micologia. Universidade Federal de Brasília. Associação Latino Americana de Micologia, Brasília, México, p.139-147.

CAVALCANTI, L.H. \&ARAÚJO, V.L.F. 1985. Myxomycetes da Paraíba II. Liceales. In Anais da VIII Reunião Nordestina de Botânica, Sociedade Botânica do Brasil Seccional de Pernambuco, Recife, p.193-198.

CAVALCANTI, L.H. \& FORTES, S.T. 1995. Myxomycetes do Estado de Santa Catarina, Brasil. Broteria 67:23-35.

CAVALCANTI, L.H. \& MARINHO, M.G.V. 1985. Myxomycetes da Paraíba I. Trichiales. In Anais da VIII Reunião Nordestina de Botânica, Recife. Sociedade Botânica do Brasil Seccional de Pernambuco, Recife, p.185-191.

CAVALCANTI, L.H. \& OLIVEIRA, F.C. 1985. Myxomycetes da Paraíba IV. Stemonitales. In Anais da VIII Reunião Nordestina de Botânica, Recife. Sociedade Botânica do Brasil Seccional de Pernambuco, Recife, p.207-214.

CAVALCANTI, L.H. \& PUTZKE, J. 1998. Myxomycetes da Chapada do Araripe (Crato-CE). Acta Botanica Brasilica 12:257-265.

CAVALCANTI, L.H. \& SILVA, J.V.B. 1985. Myxomycetes da Paraíba III. Physarales. In Anais da VIII Reunião Nordestina de Botânica. Recife, Sociedade Botânica do Brasil Seccional de Pernambuco, Recife, p.199-205.

CAVALCANTI, L.H., SANTOS, E.J. \& GOMES, N.A. 1999. Myxomycetes do estado de Roraima, com especial referência para a Estação Ecológica de Maracá (AmajariRR, Brasil). Acta Amazônica 29:195-200.

CAVALCANTI, L.H., SANTOS, E.J., SILVA, M.I.L. \& PINTO, I.M.A. 1985. Myxomycetes em cana-de-açúcar. In Anais da VIII Reunião Nordestina de Botânica, Recife. Sociedade Botânica do Brasil Seccional de Pernambuco, Recife, p.215-221.

CAVALCANTI, L.H., TAVARES, H.F.M., NUNES, A.T. \& SILVA, C.F. 2006a. Mixomicetos In Diversidade e conservação da Floresta Atlântica ao Norte do Rio São Francisco. (K.L. Porto, J.S. Almeida-Cortês \& M. Tabarelli, orgs.). Ministério do Meio Ambiente, Brasília. p.53-74.

CAVALCANTI, L.H., PONTE, M.P.P. \& MOBIN, M. 2006b. Myxomycetes, State of Piauí, Northeast Brazil. Check List 2:70-74.

FARR, M.L. 1960. The Myxomycetes of the IMUR Herbarium with special reference to Brazilian species. Publicação do Instituto de Micologia 184:1-54.
FARR, M.L. 1976. Myxomycetes. Flora Neotropica. Monograph 16. New York Botanical Garden, New York

FARR, M.L. \& KELLER, H.W. 1982. Family Elaeomyxaceae (Myxomycetes) validated. Mycologia 74:857-858.

GOTTSBERGER, G. 1968. Myxomyceten aus Bahia und Goiás. Nova Hedwigia 15:361-368.

GOTTSBERGER, G., SCHMIDT, I. \& MEIJER, A.R. 1992. Myxomycetes from the state of Paraná-Brasil 2. Arquivos de Biologia e Tecnologia 33:631-633.

HAGELSTEIN, R. 1942. A new genus of the Mycetozoa. Mycologia 34:593-594.

HOCHGESAND, E. \& GOTTSBERGER, G. 1996. Myxomycetes from the state of São Paulo, Brazil. Boletim do Instituto de Botânica 10:1-46.

JAHN, E. 1904. Myxomyceten aus Amazonas. Hedwigia 43:300-305.

KELLER, H.W. 1980. Corticicolous Myxomycetes VIII: Trabrooksia, a new genus. Mycologia 72:395-403.

LADO, C. 2001. Nomenmyx - A nomenclatural taxabase of Myxomycetes. Cuadernos de Trabajo de Flora Micológica Ibérica 16. Consejo Superior de Investigaciones Científicas Real Jardín Botânico, Madrid.

LADO, C. 2005-2010. An on line nomenclatural information system of Eumycetozoa. http://www.nomen. eumycetozoa.com (acesso em 16/03/2011).

MAIMONI-RODELLA, R.C. 2002. Biodiversidade e distribuição de mixomicetos em ambientes naturais e antropogênicos no Brasil: espécies ocorrentes nas Regiões Sudeste e Centro-Oeste. In Biodiversidade, conservação e uso sustentável da flora do Brasil. (E.L.L.A. Araújo, A.N. Moura, E.V.S.B. Sampaio, L.M.S. Gestinari \& J.M.T. Carneiro, eds.). Universidade Federal Rural de Pernambuco, Sociedade Botânica do Brasil, Brasília, p.217-220.

MARTIN, G.W. \& ALEXOPOULOS, C.J. 1969. The Myxomycetes. University of Iowa Press, Iowa City.

MARTIN, G.W., ALEXOPOULOS, C.J. \& FARR, M.L. 1983. The genera of Myxomycetes. University of Iowa Press, Iowa City.

MAYO, S.J. \& FEVEREIRO. V.P.B. 1982. Mata do PauFerro, a pilot study of the brejo forest of Paraiba, Brazil. Royal Botanic Gardens, Kew.

MOBIN, M. \& CAVALCANTI, L.H. 1999. Physarales (Myxomycetes) do Parque Nacional de Sete Cidades (Piauí, Brasil). Hoehnea 26:1-14.

NANNENGA-BREMEKAMP, N.E. 1991. A guide to temperated Myxomycetes. Biopress Limited, Bristol.

PUTZKE, J. 1996. Myxomycetes no Brasil. Cadernos de Pesquisa, Série Botânica 8:1-133.

PUTZKE, J. 2002. Myxomycetes na Região Sul do Brasil. In Biodiversidade, conservação e uso sustentável da flora do Brasil. (E.L.L.A. Araújo, A.N. Moura, E.V.S.B. Sampaio, L.M.S. Gestinari \& J.M.T. Carneiro, eds.). Universidade Federal Rural de Pernambuco, Sociedade Botânica do Brasil, Brasília, p.221-223. 
RODRIGUES, C.L.M. \& GUERRERO, R.T. 1990. Myxomycetes do Morro Santana, Porto Alegre, Rio Grande do Sul. Boletim do Instituto de Biociências 46:1-102.

SANTOS, E.J. \& CAVALCANTI, L.H. 1988. Revisão de Myxomycetes ocorrentes em cana-de-açúcar (Saccarum spp.). Boletín Micológico 4:61-64.

SANTOS, E.J. \& CAVALCANTI L. H. 1991. Myxomycetes do Canavial I. Levantamento florístico em Carpina, Pernambuco. Acta Botanica Brasilica 5:53-61.

SCHNITTLER, M. \& STEPHENSON, S. 2002. Inflorescences of Neotropical herbs as a newly discovered microhabitat for Myxomycetes. Mycologia 94:6-20.
SILVA, G.S. \& BEZERRA, J.L. 2005. Ocorrência de Fuligo septica em alface e coentro de caboclo. Fitopalogia Brasileira 30:439-442.

SPEGAZZINI, C. 1919. Reliquiae mycologicae tropicae et fungi Costaricenses nonnulli. Boletin de la Academia Nacional de Ciências de Córdoba 23: 365-609.

SYDOW, H. \& SYDOW, P. 1907. Verzeichinis der von Herrn F. Noack in Brasilien gesammelten Pilze. Annales Mycologici 5:348-363.

TORREND, C. 1915. Myxomycetes du Brésil, connus jusqu'ici. Broteria 13:72-88. 
